\title{
Formation Mechanism for a Hybrid Supramolecular Network Involving Cooperative Interactions
}

\author{
Manuela Mura, ${ }^{1}$ Fabien Silly, ${ }^{2}$ Victor Burlakov, ${ }^{3}$ Martin R. Castell, ${ }^{4}$ G. Andrew D. Briggs, ${ }^{4}$ and Lev N. Kantorovich ${ }^{1}$ \\ ${ }^{1}$ Physics, King's College London, The Strand, London, WC2R 2LS, United Kingdom \\ ${ }^{2}$ Department of Materials, University of Oxford, Parks Road, Oxford OX1 3PH, United Kingdom; CEA, IRAMIS, SPCSI, Hybrid \\ Magnetic Nanoarchitectures, F-91191 Gif-sur-Yvette, France \\ ${ }^{3}$ OCCAM, Mathematical Institute, Oxford University, 24-29 St Giles, Oxford, OX1 3LB, United Kingdom \\ ${ }^{4}$ Department of Materials, University of Oxford, Parks Road, Oxford OX1 3PH, United Kingdom
}

(Received 21 January 2012; published 27 April 2012)

\begin{abstract}
A novel mechanism of hybrid assembly of molecules on surfaces is proposed stemming from interactions between molecules and on-surface metal atoms which eventually got trapped inside the network pores. Based on state-of-the-art theoretical calculations, we find that the new mechanism relies on formation of molecule-metal atom pairs which, together with molecules themselves, participate in the assembly growth. Most remarkably, the dissociation of pairs is facilitated by a cooperative interaction involving many molecules. This new mechanism is illustrated on a low coverage Melamine hexagonal network on the $\mathrm{Au}(111)$ surface where multiple events of gold atoms trapping via a set of so-called "gate" transitions are found by kinetic Monte Carlo simulations based on transition rates obtained using ab initio density functional theory calculations and the nudged elastic band method. Simulated STM images of gold atoms trapped in the pores of the Melamine network predict that the atoms should appear as bright spots inside Melamine hexagons. No trapping was found at large Melamine coverages, however. These predictions have been supported by preliminary STM experiments which show bright spots inside Melamine hexagons at low Melamine coverages, while empty pores are mostly observed at large coverages. Therefore, we suggest that bright spots sometimes observed in the pores of molecular assemblies on metal surfaces may be attributed to trapped substrate metal atoms. We believe that this type of mechanism could be used for delivering adatom species of desired functionality (e.g., magnetic) into the pores of hydrogen-bonded networks serving as templates for their capture.
\end{abstract}

DOI: 10.1103/PhysRevLett.108.176103

PACS numbers: 68.43.Mn

Recent STM studies of hydrogen-bonded molecular assemblies on crystal surfaces have resulted in significant progress in our ability to create and modify these selforganized networks [1-10]. The motivation for much of this research is the premise that control of the porous network structure can be achieved by modifying the component molecules which may result in changes to the size and shapes of the pores $[4,11]$. The pores can be used as a template to host foreign species of required functionality [12]. However, this template approach has intrinsic limitations due to the strict requirements concerning the relative sizes of the pores and guest species. Violation of these requirements may result in defects, such as more than one functional unit per pore, which might compromise the performance of the network. For this reason it is extremely important to develop alternative kinetic pathways, which ensure defect-free fabrication of functional surface networks.

In this Letter we describe such a kinetic pathway based on the co-assembly of a network constructed from an organic molecule and an atomic species. We show that there are two key factors in this process that ensure delivery of no more than one atom per pore. First, atomic-molecular dimers need to be formed to take part in the network formation. Second, there is a requirement of a specific gating mechanism of atomic insertion into a pore mediated by collective interactions between the network molecules and atoms. The important factor here is the existence of freely diffusing native metal substrate adatoms. The free metal adatoms may play an important role in the formation of adlayers on surfaces [13]; however, the relevance of this prediction for molecular assembly is rarely discussed. At variance with metal coordination phenomena $[4,14-19]$, we propose a new mechanism whereby metal atoms may directly participate in the molecular assembly without affecting the bonding between the molecules. We illustrate this mechanism on a prototype system of the hexagonal network of Melamine molecules, Figs. 1(a) $-1(d)$, on the $A u(111)$ surface [20-24] which may trap individual gold atoms. Our study is mainly theoretical, but the results are supported by experimental STM images.

The calculations were performed using the $a b$ initio density functional theory (DFT) SIESTA method [25,26] using the Perdew, Becke, and Ernzerhof (PBE) [27] density functional with semiempirical dispersion interaction [28], and a two layer gold slab [29]. As the Melamine molecules adsorb on the surface, they start interacting with mobile $\mathrm{Au}$ adatoms. Calculations suggest that it is energetically 


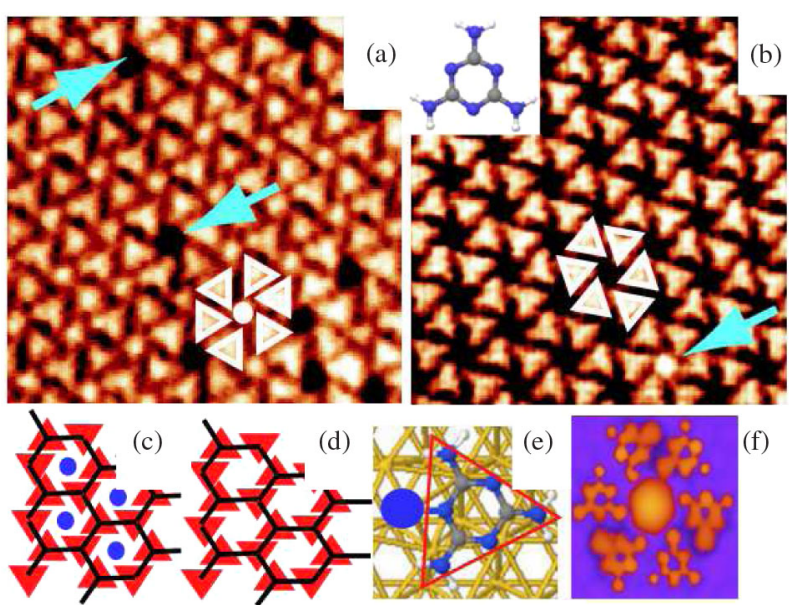

FIG. 1 (color online). High resolution STM images of (a) an island after deposition of $0.1 \mathrm{ML}\left(6 \times 6 \mathrm{~nm}^{2}\right.$ area; $V_{s}=$ $\left.-0.6 \mathrm{~V}, I_{t}=0.1 \mathrm{nA}\right)$ and (b) $1 \mathrm{ML}\left(6 \times 6 \mathrm{~nm}^{2}\right.$ area; $V_{s}=$ $-1.8 \mathrm{~V}, I_{t}=0.5 \mathrm{nA}$ ) of Melamine [shown in the inset of (b) with grey, blue and white colors for $\mathrm{C}, \mathrm{N}$, and $\mathrm{H}$ atoms] on $\mathrm{Au}$ (111)- $(22 \times \sqrt{3})$ surface. On both images one can clearly see hexagonal lattices formed by triangles representing individual molecules (indicated as white). In (a) a large number of bright spots is found inside hexagons of Melamines, while in (b) these are rare. Arrows in (a) and (b) indicate occasional dark and bright spots, respectively. Schematic presentations of the two structures (a) and (b) are shown in (c) and (d) with Melamine molecules depicted as red triangles, and in (c) bright spots as blue circles. (e) A stable $\mathrm{M}+\mathrm{Au}$ pair formed by Melamine (indicated with a red triangle) and $\mathrm{Au}$ atom (large blue circle). (f) Calculated STM image of a Melamine hexagon with a $\mathrm{Au}$ atom inside on the gold surface.

favorable (by up to $0.9 \mathrm{eV}$ ) for a Melamine molecule to trap a single Au adatom whereby forming a stable $M+$ Au pair, Fig. 1(e). The molecules and pairs were found to be quite mobile at room temperature (RT) (the diffusion barriers are $\simeq 0.05$ and $0.2 \mathrm{eV}$, respectively [29]). Hence, molecules will readily find free adatoms and form pairs; as the concentration of the adatoms is reduced, the thermal equilibrium with step edges is shifted and more adatoms become available, i.e., Melamine molecules would mop up all available $\mathrm{Au}$ atoms and then serve as their carriers across the surface. Note that only Au atoms attached alone to a step edge have the lowest barrier to detach from it $(\simeq 0.43 \mathrm{eV}[29])$.

Importantly, another Melamine molecule can only attach to the $\mathrm{M}+\mathrm{Au}$ pair at either of the two sides not already occupied by the Au atom (with the energy gain of $0.42 \mathrm{eV}$ [22-24]). This should affect the formation of the Melamine network in which Melamine molecules attach to all three sides of every molecule whereby building a hexagonal structure [20-24], see Fig. 1(d). We find that the high energy barrier of $0.9 \mathrm{eV}$ for the dissociation of a single $\mathrm{M}+$ Au pair, unsurmountable at RT, is greatly reduced (to $0.2-0.4 \mathrm{eV}$ ) when four molecules are attached to the pair as shown in Fig. 2(a) forming an incomplete hexagon with a
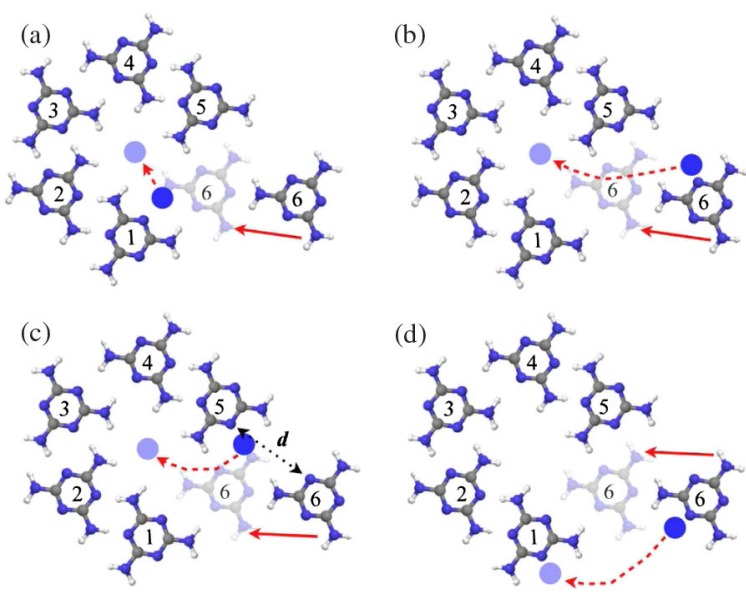

FIG. 2 (color online). Schematics of four investigated gate transitions. The $\mathrm{Au}$ atom (larger blue circle) and the sixth molecule coming to close the hexagon are shown in full color or as dim in their initial and final configurations, respectively. The $\mathrm{Au}$ atom goes into the hexagon center if: (a) it is on the inner side of molecule 1; (b) when the $\mathrm{M}+\mathrm{Au}$ pair approaches the pentagon at the gate site as shown; (c) the Au atom is attached to the outer side of molecule 5 when it initially jumps on the side of molecule 6 facing the gate (the barrier for this elementary transition depends on distance $d$ between $\mathrm{N}$ atoms of molecules 5 and 6), and then goes into the hexagon center while molecule 6 closes the gate, exactly as in (b). Finally, a different orientation of the $\mathrm{M}+\mathrm{Au}$ pair results in the Au atom ending up on the outer side of molecule 1 in (d).

"gate" between the molecules 1 and 5 (the "cooperativity" effect). Hence, the self-assembly process can be envisaged as a sequence of Melamine to Melamine attachment events accompanied by the pairs dissociation; the detached $\mathrm{Au}$ atoms either diffuse out of the forming hexagon to find themselves trapped outside it, or become trapped inside the hexagons during their closure by the incoming sixth molecule. Moreover, our calculations show that it is energetically favorable for $\mathrm{Au}$ adatoms to be inside hexagons [29].

Several possible gate mechanisms, shown schematically in Fig. 2, either lead to the Au atom trapping (a)-(c) or it ending up outside attached to molecule 1 (d). However, even in the latter case the Au atom would eventually end up in the center of the adjacent hexagon during its construction via transition (a). To calculate the corresponding barriers, we performed extensive nudged elastic band (NEB) [30,31] DFT calculations, and found that the upper boundaries for the barriers for the four transitions are $0.41,0.25,0.25$, and $0.41 \mathrm{eV}$. Preliminary analyses indicated much larger energy barriers for other transitions that can be envisaged (e.g., for the adatom in case (d) moving inside the hexagon), suggesting their contribution to the growth kinetics being marginal.

To model the growth of the network upon evaporation of Melamines on the $\mathrm{Au}(111)$ surface with a supply of free $\mathrm{Au}$ atoms, we performed extensive RT kinetic Monte Carlo 
(KMC) simulations. Although the concentration of free adatoms can be evaluated knowing the relevant energy barriers and the surface structure using, e.g., steps kinetics models [13,32], this goes beyond the scope of this study, and we accepted adatom concentration as a parameter of the theory.

We considered a $80 \times 80$ simulation region with the hexagonal grid. Melamine molecules were allowed to occupy every site of the grid apart from the hexagon centers. A certain number of gold atoms were added at the start of the simulations which were allowed to occupy all sites including the hexagon centers. No periodic boundary conditions were used. Instead, the simulation region was surrounded by a running "wall" mimicking the step edges. Melamine molecules were not allowed to approach the walls, while the gold atoms were able to attach to it without any barrier, and also detach with the barrier of $0.43 \mathrm{eV}$. Free $\mathrm{Au}$ atoms and Melamine molecules diffuse on the surface with the calculated barriers of 0.1 and $0.05 \mathrm{eV}$, respectively. Melamine molecules can rotate by $60^{\circ}$ on the same lattice site with the same diffusion barrier. Au atoms were allowed to attach (detach) to (from) Melamine molecules (by no more than a single Au atom allowed to attach), and the barrier of $0.9 \mathrm{eV}$ was applied to the detachment transition. The created $\mathrm{M}+$ Au pairs are able to diffuse on the hexagonal lattice and rotate by $60^{\circ}$ with the calculated barrier of $0.2 \mathrm{eV}$. Melamine molecules can also attach/ detach to/from each other with the detachment barrier of 0.42 or $0.84 \mathrm{eV}$ corresponding to one and two neighbors, respectively, $(0.42 \mathrm{eV}$ is the Melamine dimer binding energy [23,24]). Clusters of molecules were assumed to be immobile, although, as described above, single molecules are able to detach from clusters into nearest empty sites. All four gate transitions were also implemented, with the barriers chosen to be by $\simeq 0.1 \mathrm{eV}$ smaller than the NEB calculated values. This is due to the fact that the calculated barriers may only serve as upper boundaries [29]. Note that for the gate transition to happen, only three molecules are needed: two at the gate and one coming to it, and hence a successful 'gate' transition does not yet guarantee the capture of the $\mathrm{Au}$ atom as it may escape (diffuse out) if the hexagon is not yet complete. All preexponential factors in the rates were taken to be $10^{13} \mathrm{~s}^{-1}$.

Initially, we placed a certain number of Au atoms on the hexagonal lattice (surrounded by the wall) and equilibrated the system. This required nanosecond time scales and resulted in most of the $\mathrm{Au}$ atoms decorating the wall (step edges) and a rather small number of them (on average) distributed across the lattice at any single time. Then deposition of the Melamine molecules was initiated with a predefined constant rate and the KMC simulation was allowed to run much longer than the desired Melamine coverage was reached. As Melamine molecules are deposited, they start forming $\mathrm{M}+$ Au pairs whereby displacing the thermodynamic equilibrium between the free Au atoms on the lattice and those attached to the wall, so that more Au atoms detach from the wall and become available to the Melamines; this continues until the whole supply of the $\mathrm{Au}$ atoms is exhausted and all of them are found either trapped within the Melamine hexagons or decorating the islands edges. We find that most of the Au atoms decorate the edges of Melamine islands, however, as shown in Fig. 3 (insets), their considerable number (about 10\%-15\%) are found trapped inside hexagons for some $\mathrm{Au}$ atoms concentrations.

Typical time dynamics of the number of Melamine molecules deposited on the surface, $\mathrm{M}+\mathrm{Au}$ pairs, $\mathrm{Au}$ atoms attached to the wall and trapped inside Melamine hexagons are shown in Fig. 3. One can clearly see a gradual decrease of the $\mathrm{Au}$ atoms at the wall strongly correlated with the formation of $M+A u$ pairs. Initially, the number of $\mathrm{M}+\mathrm{Au}$ pairs increases in the same way as the number of deposited Melamine molecules; however, after the point $A$, the number of molecules arriving on the surface is larger than the number of $\mathrm{Au}$ atoms becoming available, and the increase of the $\mathrm{M}+\mathrm{Au}$ pairs slows down. After the point $B$, no more $\mathrm{Au}$ atoms are available and the process of formation of new $\mathrm{M}+$ Au pairs stopped. After that instant, one can observe the growth in the number of the Au atoms trapped in the Melamine hexagons; at the same time the number of $\mathrm{M}+\mathrm{Au}$ pairs is reduced, and, as one would expect, both events are strongly correlated. Note that, if both the $\mathrm{M}+\mathrm{Au}$ pairs formation and the Au atoms deposition from the wall happen rather quickly, trapping of $\mathrm{Au}$ atoms inside hexagons takes a very long time (seconds) and

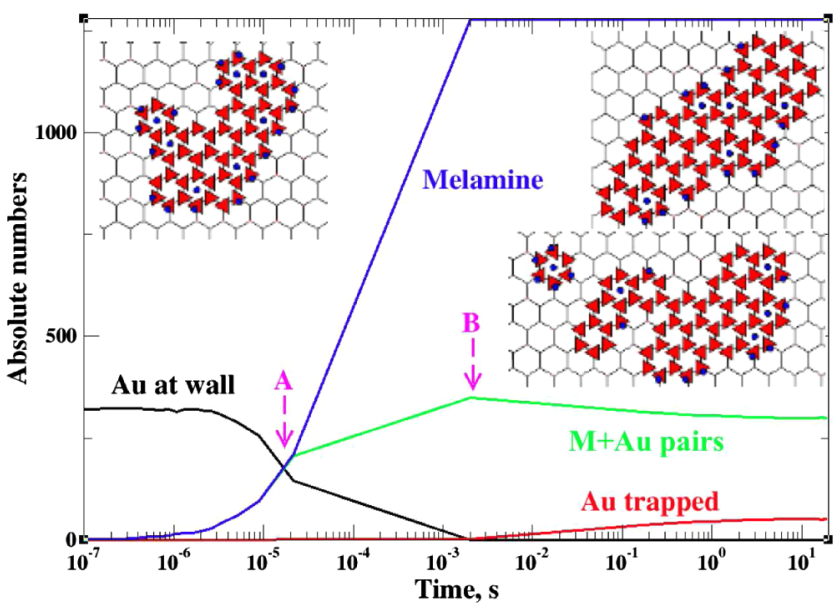

FIG. 3 (color online). Time dynamics of the number of deposited Melamine molecules (blue), formed $\mathrm{M}+\mathrm{Au}$ pairs (green), $\mathrm{Au}$ atoms attached to the wall (black) and trapped inside Melamine hexagons (red) during a KMC simulation. Typical Melamine islands stabilized after a KMC run and shown in insets are seen to contain trapped $\mathrm{Au}$ atoms (blue) and decorated edges. The density of trapped $\mathrm{Au}$ atoms varies from one island to the other. Calculation details: deposition rate $10^{-5} \mathrm{ps}^{-1}$, Melamine coverage 0.1 , initial concentration of $\mathrm{Au}$ atoms 0.018 . 
most of the processes happen after the Melamine coverage has been reached. We stress that gate transitions were competing in our KMC simulations with pure diffusion of Au adatoms, and all successful trapping events occurred entirely due to the former mechanism with all four transitions contributed practically equally.

The results presented above correspond to a rather low Melamine coverage of $0.1 \mathrm{ML}$. We have also performed similar calculations for higher coverages of up to $0.4 \mathrm{ML}$ using a range of deposition rates between $10^{-5}$ and $10^{-3}$ (which are still many orders of magnitude faster than in experiments). We find that the number of trapped Au atoms depends rather weakly on the deposition rate. However, it strongly depends on the Melamine coverage: at higher coverages above $0.2 \mathrm{ML}$ the number of trapped Au atoms is substantially reduced as compared with the case of 0.1 ML coverage. This is explained by the fact that at high Melamine coverages pure Melamine islands are built much faster than the gate transitions are capable placing the $\mathrm{Au}$ atoms into hexagon centers during the islands growth leading mostly to decoration of the islands with the Au atoms. Summarizing, our calculations predict that at low Melamine coverages a considerable number of $\mathrm{Au}$ atoms get trapped in the pores of the Melamine hexagonal network; at high coverages the trapping is much less frequent. For any coverage we also find that islands edges also get decorated with Au atoms.

If $\mathrm{Au}$ atoms are able to occupy pores of the Melamine hexagonal network, they may be seen in STM images, and indeed, when we calculated the STM images of a Melamine hexagon on the gold surface with and without a gold atom in its center [29], only in the former case do we find a bright spot in it, Fig. 1(f). These findings have been evaluated in our STM experiments in which we imaged Melamine networks at low and high coverages at RT in an STM operating at a pressure of $10^{-8} \mathrm{~Pa}$. Melamine molecules were sublimated at $100^{\circ} \mathrm{C}$. Etched tungsten tips were used to obtain constant current images at RT with a bias voltage applied to the sample. The network structure as seen in STM images was found to depend mostly on the coverage; no differences were found when several flux values for Melamine deposition were applied. High resolution STM images after deposition of Melamine molecules on the $\mathrm{Au}(111)$ surface are shown in Figs. 1(a) and 1(b). The high (1 ML) coverage image (b) reveals the molecules forming a hexagonal lattice with dark spots in the center of most hexagons composed of six molecules, the latter appearing in the images as triangular protrusions [20-24]. Only few bright spots in Melamine hexagons are visible (indicated by arrows). In comparison, most of the Melamine hexagons are filled with a bright spot each at low (0.1 ML) coverage shown in (a), with only few dark spots clearly visible inside some hexagons, also indicated by arrows (large scale STM images are provided in SI). Note that the boundaries of Melamine islands with trapped
Au species appear blurry in our STM images [29], so that we were unable to verify decoration of islands boundaries with $\mathrm{Au}$ atoms as predicted by theory.

Bright spots in the pores of the networks of organic molecules on metal surfaces were observed previously. In [33] these were assigned to Melamine molecules standing upright in the pores of their hexagons. In [34] rare bright spots were observed in the PTCDA network on the $\mathrm{Ag}$ (110) surface after $\mathrm{Ag}$ atoms deposition; clearly, these extra features were attributed to the $\mathrm{Ag}$ adatoms. Our findings seem to indicate that the bright spots observed in [33] correspond to the Au atoms trapped during the network formation. The hypothesis associating bright spots with upright Melamine molecules [33] seems unlikely as it would favor bright spots to exist both at low and high coverages which contradicts both our observations and theoretical predictions.

One may think that bright spots may be related to the herringbone reconstruction (HR) of the $\mathrm{Au}(111)$ surface itself, which "expels" extra atoms (responsible for the strain in the upper gold layer [35]) into the pores of the hexagons during the assembly growth. This is unlikely since at high coverages the characteristic HR is visible as imprinted on the Melamine network, i.e., is not lifted $[22,29]$. Penetration of $\mathrm{Au}$ atoms into the pores of an existing Melamine network should also be ruled out due to unsurmountable energy barriers at RT. One is left with the explanation proposed here. It is possible that some other mechanisms may also exist; however, it is clear, due to rather small transition barriers we calculated, that the gate transitions must be playing a very important, if not dominant, role in the Au atoms trapping.

Concluding, we have found a new kinetic pathway for generating functionalized surface nanostructures based on specific mechanisms of embedding functionalizing atoms into porous molecular networks. Using melamine on $\mathrm{Au}(111)$ as an example, we show that the key feature of this pathway is the formation of melamine-Au pairs. The latter directly participate in the formation of hexagonal melamine network and provide functionalizing $\mathrm{Au}$ atoms into the pores via specific gating mechanisms mediated by cooperative molecular interactions. The trapped Au atoms manifest themselves as bright spots which we observed in our STM experiments. The proposed mechanism we believe is not only restricted to the Melamine-gold system. This is supported by our preliminary calculations of other molecules on gold [29] which demonstrate atom-molecule pair formation. We hope that the present work will stimulate more studies leading to better understanding of the role played by native surface adatoms in formation of molecular assemblies in this and other systems for which the Melamine-gold system may serve as a prototype example.

The authors thank the EPSRC (EP/D048761/1 and GR/ S15808/01) for funding, Chris Spencer (JEOL UK) for 
valuable technical support and Philip Moriarty for helpful discussions. F.S. also thanks the European Research Council (FP7/2007-2013)/ERC grant agreement No. 259297 for funding.

*lev.kantorovitch@kcl.ac.uk

[1] G. Pawin, K.L. Wong, K. Y. Kwon, and L. Bartels, Science 313, 961 (2006).

[2] J. V. Barth, Annu. Rev. Phys. Chem. 58, 375 (2007).

[3] R. Otero et al., Science 319, 312 (2008).

[4] J. V. Barth, Surf. Sci. 603, 1533 (2009).

[5] K. Bishop, C. Wilmer, S. Soh, and B. Grzybowski, Small 5, 1600 (2009).

[6] F. Besenbacher, J. Lauritsen, and T. Linderoth, Surf. Sci. 603, 1315 (2009).

[7] R Raval, Chem. Soc. Rev. 38, 707 (2009).

[8] S.-C. Li, L.-N. Chu, X.-Q. Gong, and U. Diebold, Science 328, 882 (2010).

[9] K. J. Franke, G. Schulze, and J. L. Pascual, Science 332, 940 (2011).

[10] M. Kim et al., Science 331, 1312 (2011).

[11] D. E. Hooks, T. Fritz, and M. D. Ward, Adv. Mater. 13, 227 (2001).

[12] J. A. Theobald, N.S. Oxtoby, M.A. Phillips, N.R. Champness, and P.H. Beton, Nature (London) 424, 1029 (2003).

[13] W. W. Pai, N. C. Bartelt, and J. E. Reutt-Robey, Phys. Rev. B 53, 15991 (1996).

[14] P. Messina et al., J. Am. Chem. Soc. 124, 14000 (2002).

[15] A. Dmitriev, H. Spillmann, N. Lin, J. V. Barth, and K. Kern, Angew. Chem., Int. Ed. Engl. 42, 2670 (2003).

[16] T. Classen et al., Angew. Chem., Int. Ed. Engl. 44, 6142 (2005).
[17] U. Schlickum et al., Nano Lett. 7, 3813 (2007).

[18] M. Matena, T. Riehm, M. Stöhr, T. A. Jung, and L. H. Gade, Angew. Chem., Int. Ed. Engl. 47, 2414 (2008).

[19] N. Lin, S. Stepanow, M. Ruben, and J. V. Barth, Topics in Current Chemistry, Topics in Current Chemistry Vol. 287 (Springer, Berlin, Heidelberg, 2008).

[20] L. M. A. Perdigao et al., J. Phys. Chem. B 110, 12539 (2006).

[21] W. Xu et al., Small 3, 854 (2007).

[22] F. Silly et al., J. Phys. Chem. C 112, 11476 (2008).

[23] M. Mura, N. Martsinovich, and L Kantorovich, Nanotechnology 19, 465704 (2008).

[24] M. Mura, A. Gulans, T. Thonhauser, and L. Kantorovich, Phys. Chem. Chem. Phys. 12, 4759 (2010).

[25] D. Sanchez-Portal, P. Ordejon, E. Artacho, and J. M. Soler, Int. J. Quantum Chem. 65, 453 (1997).

[26] J. M. Soler et al., J. Phys. Condens. Matter 14, 2745 (2002).

[27] J. P. Perdew, K. Burke, and M. Ernzerhof, Phys. Rev. Lett. 77, 3865 (1996).

[28] S. Piana and A. Bilic, J. Phys. Chem. B 110, 23467 (2006).

[29] See Supplemental Material at http://link.aps.org/ supplemental/10.1103/PhysRevLett.108.176103 for further details.

[30] G. Henkelman and H. Jónsson, J. Chem. Phys. 113, 9978 (2000).

[31] G. Henkelman, B. P. Uberuaga, and H. Jónsson, J. Chem. Phys. 113, 9901 (2000).

[32] N.C. Bartelt, J.L. Goldberg, T.L. Einstein, and E.D. Williams, Surf. Sci. 273, 252 (1992).

[33] H. M. Zhang et al., J. Phys. Chem. C 112, 4209 (2008).

[34] M. Böhringer, W. D. Schneider, K. Glöckler, E. Umbach, and R. Berndt, Surf. Sci. 419, L95 (1998).

[35] Y. Wang, N. Hush, and J. Reimers, Phys. Rev. B 75, 233416 (2007). 